\title{
JWST science instrument pupil alignment measurements
}

\author{
Dave Kubalak $^{\mathrm{a}}$, Joe Sullivan ${ }^{\mathrm{b}}$, Ray Ohl ${ }^{\mathrm{a}}$, Scott Antonille ${ }^{\mathrm{a}}$, Alexander Beaton ${ }^{\mathrm{c}}$, Phillip Coulter ${ }^{\mathrm{a}}$, \\ George Hartig ${ }^{\mathrm{d}}$, Doug Kelly ${ }^{\mathrm{e}}$, David Lee ${ }^{\mathrm{f}}$, Michael Maszkiewicz ${ }^{\mathrm{g}}$, Paul Schweiger ${ }^{\mathrm{h}}$, Randal Telfer ${ }^{\mathrm{d}}$, \\ Maurice Te Plate ${ }^{i}$, Martyn Wells ${ }^{\mathrm{f}}$ \\ aNASA Goddard Space Flight Center, Greenbelt, Maryland, USA 20771 \\ bBall Aerospace \& Technologies Corp, 1600 Commerce Street, Boulder, Colorado, USA 80306 \\ 'ComDev International, 303 Terry Fox Drive Suite 100, Ottawa On K2K 3J1 Canada \\ ${ }^{\mathrm{d} S}$ Space Telescope Science Institute, 3700 San Martin Drive, Baltimore, MD 21218 \\ eUniversity of Arizona, Tucson AZ 85721 \\ fUK Astronomy Technology Centre, Royal Observatory Edinburgh \\ 'Canadian Space Agency,6767, route de l'Aéroport, Saint-Hubert, Québec J3Y 8Y9 Canada \\ hLockheed Martin Space Systems Co. (United States) \\ iEuropean Space Agency, Keplerlaan 1, PO Box 299, 2200 AG, Noordwijk, The Netherlands
}

\begin{abstract}
NASA's James Webb Space Telescope (JWST) is a 6.5m diameter, segmented, deployable telescope for cryogenic IR space astronomy ( 40K). The JWST Observatory architecture includes the Optical Telescope Element (OTE) and the Integrated Science Instrument Module (ISIM) element that contains four science instruments (SI), including a guider. OSIM is a full field, cryogenic, optical simulator of the JWST OTE. It is the "Master Tool" for verifying the cryogenic alignment and optical performance of ISIM by providing simulated point source/star images to each of the four Science Instruments in ISIM. Included in OSIM is a Pupil Imaging Module (PIM) - a large format CCD used for measuring pupil alignment. Located at a virtual stop location within OSIM, the PIM records superimposed shadow images of pupil alignment reference (PAR) targets located in the OSIM and SI pupils.
\end{abstract}

The OSIM Pupil Imaging Module was described by Brent Bos, et al, at SPIE in 2011 prior to ISIM testing. We have recently completed the third and final ISIM cryogenic performance verification test before ISIM was integrated with the OTE. In this paper, we describe PIM implementation, performance, and measurement results.

Keywords: JWST, ISIM, pupil alignment, pupil, pupil shear, testing, verification

\section{INTRODUCTION}

The Integrated Science Instrument Module (ISIM) of the James Webb Space Telescope (JWST) contains four Science Instruments (SI's) and the Fine Guidance Sensor (FGS). Along with other common alignment considerations such as wave front error, field of view, etc., pupil alignment is of critical importance to JWST not only to prevent vignetting, but also to suppress a potential stray light path due to the open architecture of the telescope design ${ }^{1}$. Bos et $\mathrm{al}^{2} \mathrm{described}$ various approaches to measuring ISIM pupil shear in-situ during cryogenic vacuum testing. This paper focuses on one of those methods - using Pupil Alignment References (PAR's) in the Optical Telescope Element Simulator (OSIM), and in the pupil planes of each SI. This topic is also discussed by Maszkiewicz et $\mathrm{al}^{3}$ and Rohrbach et $\mathrm{al}^{4}$.

The maximum allowable amount of lateral pupil shear at the ISIM level is $3.1 \%$ of the pupil diameter, including uncertainty. The maximum roll (clocking) allowable is $\pm 10.6 \mathrm{mR}$.

\section{METHODOLOGY}

In its simplest form, the pupil alignment test uses light from OSIM that is projected into and reflected back out of each instrument to measure misalignments between OSIM and SI PAR targets. The PAR target in OSIM is a transmissive

Optical System Alignment, Tolerancing, and Verification X, edited by José Sasián,

Richard N. Youngworth, Proc of SPIE Vol. 9951,99510D · C 2016 SPIE

CCC code: $0277-786 X / 16 / \$ 18 \cdot$ doi: $10.1117 / 12.2238830$

Proc. of SPIE Vol. 9951 99510D-1 
mask inserted into its aperture stop. As the light passes through the PAR mask, it picks up the shadow of the PAR target pattern. The light is then reflected off of Fold 3 (FM3) and directed into an instrument. The PAR targets in the SI's are reflective targets in the SI pupil planes. As light is reflected off of the SI PAR, it picks up the shadow of the SI PAR target. After reflecting off of the PAR, the light is directed back out of the SI where it proceeds towards OSIM. FM3 is a beam splitter at the wavelength used for PIM testing, so when the light returns to FM3, some of it passes through FM3 and is captured by the OSIM's Pupil Imaging Module (PIM), a large format CCD placed at a conjugate stop plane behind FM3. The image captured by the PIM will contain shadows of both the OSIM and SI PAR's. This process is shown schematically in Figure 21.

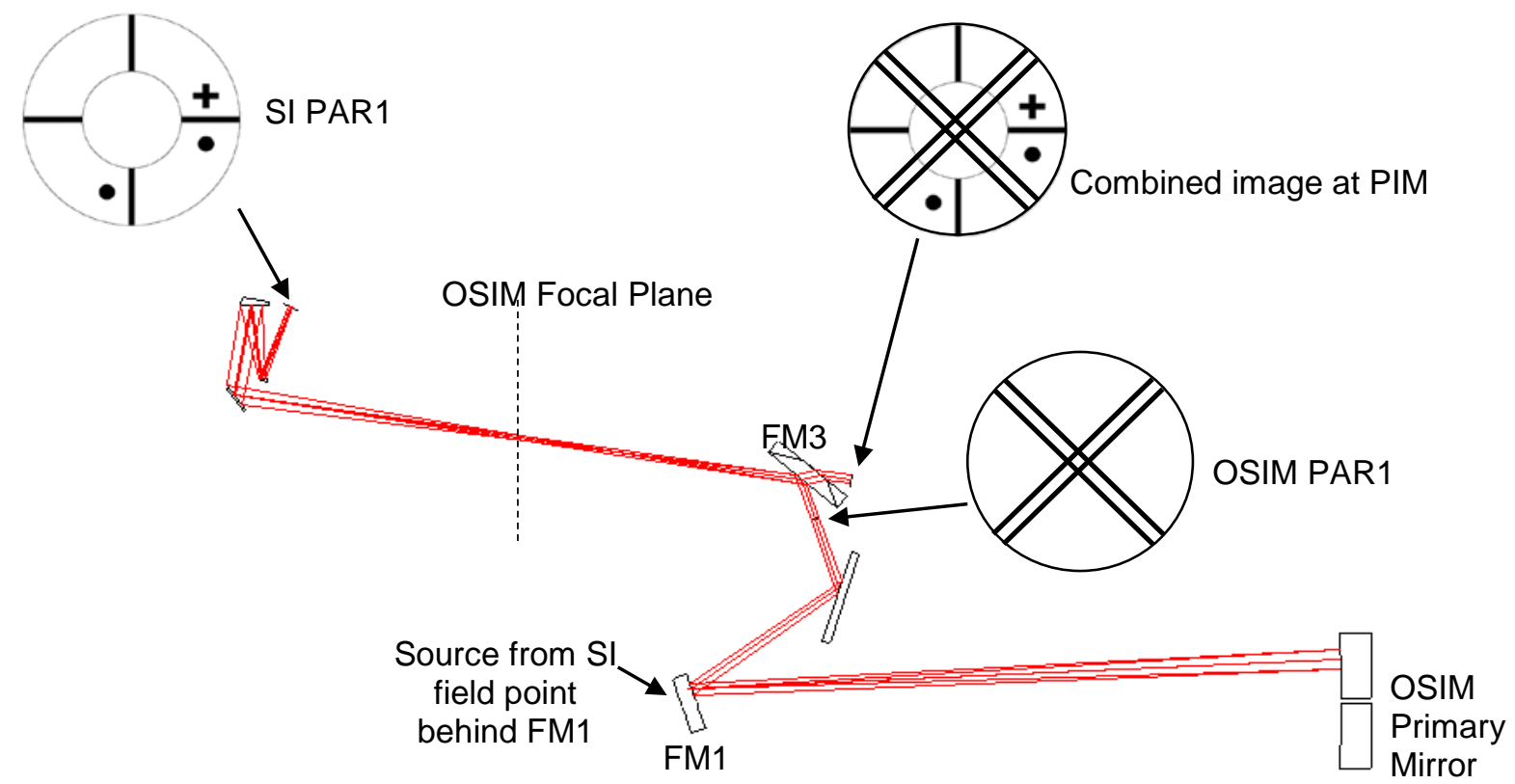

Figure 2-1 Schematic of the OSIM optical system. FGS Guider is shown in this diagram.

Offsets between PAR targets in the recorded images are compared against expected offsets, which are based on optical models and OSIM/SI calibrations. The difference between what the measured and expected offsets is reported as the final pupil shear. This document describes the OSIM and SI pupil alignment references, the pupil imaging module, and testing performed during ISIM cryo-vacuum tests CV1RR, CV2, and CV3.

\subsection{OSIM hardware}

The OSIM PAR's are held by OSIM's Pupil Select Mechanism (PSM). The PSM holds 16 aperture masks on 2 wheels, 8 masks per wheel. One mask in each wheel is a large open circle allowing access to the opposite wheel. Wheel 1, containing two OSIM Pupil Alignment References, PAR1 and PAR2, is located in the nominal pupil plane in OSIM. The aperture masks are selected by rotating the wheels until the chosen masks are in position. As the beam passes through one of the PARs, the target pattern of the PAR is imprinted as a shadow in the beam.

Drawings of the tricontagon, PAR1, and PAR2 masks are shown Figure 2-2. They are not drawn to the same scale - the PAR's are slightly larger than the central obscuration of the tricontagon. The tricontagon shape resembles the shape of the OTE mirror and is the main testing mask for OSIM. PAR1 is the primary mask for pupil alignment testing and PAR2 is the backup. Images were taken with PAR2 during testing, but the results in this paper address only PAR1. 

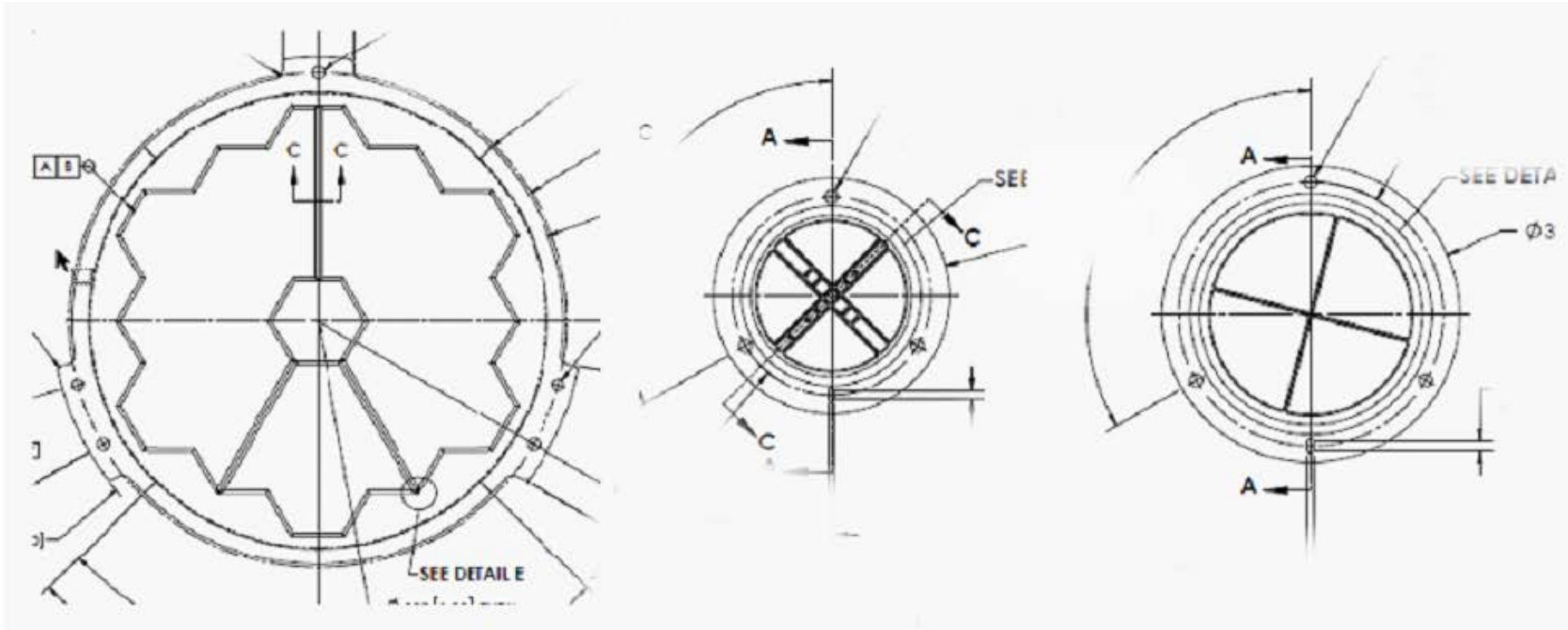

Figure 2-2 Drawings of the tricontagon, PAR1, and PAR2, respectively. The figures are not shown at the same scale.

Clearly, the relative alignment between the tricontagon and PAR's must be removed from the pupil measurement. The PSM inter-mask offset was measured in a cryogenic component-level test prior to OSIM integration. During the test, a 4-inch chamber window was used to view each mask as it was rotated into position. Within the window, alignment references were used for mask registration.

For the tricontagon measurement, a small target in the center of the mask was used to measure mask position, and the spiders were used to measure rotation. For the PAR masks, intersections of the target features were used. Table 2-1 contains the final measurement results. The tricontagon mask was used as a reference, and measurement errors associated with the tricontagon are wrapped up in the PAR mask uncertainties. Repeated measurements were used for the statistical results shown. Positions are shown in the V-coordinate system, where V2 and V3 are lateral shifts. V1 is in the focus direction.

Table 2-1 Cryogenic mask measurement results. Position is shown in the "V" coordinate system, which is based on the vertex of the primary OTE mirror. V2 and V3 are lateral directions; V1 is in the focus direction.

\begin{tabular}{|c|c|c|c|c|c|c|}
\hline & \multicolumn{3}{|c|}{ Position } & \multicolumn{3}{c|}{ 1 6 error } \\
\hline Mask & V2 (\%) & V3 (\%) & Roll (mR) & V2 (\%) & V3 (\%) & Roll (mR) \\
\hline Tricontagon & 0.00 & 0.00 & 0.00 & 0.00 & 0.00 & 0.00 \\
\hline PAR1 & 0.00 & 0.05 & 4.26 & 0.04 & 0.04 & 0.47 \\
\hline PAR2 & 0.07 & 0.03 & 3.71 & 0.00 & 0.00 & 0.29 \\
\hline
\end{tabular}

To perform a PAR measurement with a particular SI, OSIM is configured to point at that SI's field point with one of the OSIM PARs used in place of the tricontagon. Since the goal of steering OSIM is to put the pupil in the correct location, there is no additional offset due to steering - any inaccuracies are carried in uncertainties. The uncertainty associated with steering OSIM and placing the stop into the correct position is $0.11 \%(1 \sigma)$ of the pupil diameter. In addition, uncertainty in the accuracy of OSIM's calibration during pre-ISIM testing was $0.26 \%(1 \sigma)$. Therefore, the OSIM total contribution to pupil shear based on the above uncertainties and the values shown in Table 2-1 are shown in Table 2-2. The V2 and V3 uncertainties due to OSIM calibration dominate the uncertainties in the final pupil measurement. 
Table 2-2 Total OSIM contribution to expected pupil shear.

\begin{tabular}{|c|c|c|c|c|c|c|}
\hline & \multicolumn{3}{|c|}{ PAR-pupil offset } & \multicolumn{3}{c|}{ 1 $\sigma$ error } \\
\hline Item & V2 (\%) & V3 (\%) & Roll (mR) & V2 (\%) & V3 (\%) & Roll (mR) \\
\hline OSIM calibration & 0.00 & 0.00 & 0.00 & 0.26 & 0.26 & 0.00 \\
\hline OSIM repeatability & 0.00 & 0.00 & 0.00 & 0.11 & 0.11 & 0.00 \\
\hline PAR1 offset & 0.00 & 0.05 & 4.26 & 0.04 & 0.04 & 0.47 \\
\hline $\begin{array}{c}\text { Total OSIM contribution } \\
\text { (RSS of above) }\end{array}$ & 0.00 & 0.05 & 4.26 & 0.28 & 0.28 & 0.47 \\
\hline
\end{tabular}

After passing through the aperture masks in the PSM, the beam gets steered by FM3 towards ISIM. FM3 is a thick fused silica element coated to be a beam splitter between about $600--700 \mathrm{~nm}$ and a highly reflective mirror above $700 \mathrm{~nm}$. Maximum transmittance is about $40 \%$ at $632 \mathrm{~nm}$. FM3 can be tipped/tilted to point an image arbitrarily across each SI FOV. A Pupil Translation Mechanism (PTM) moves the PSM laterally to compensate for pupil motion caused by beam steering.

An additional artifact of steering OSIM this way is that it will introduce a rotation of the pupil around the chief ray (pupil clocking) if FM3 is tilted about V3. Tilting FM3 about the V2 axis causes no pupil rotation. There is no compensation for this pupil clocking, so it must be taken into account during data analysis.

Finally, when the beam returns from the SI and passes through FM3, an image is captured by the PIM. The PIM consists of a bare large-format CCD detector and supporting electronics. It is a $4 \mathrm{k} \times 4 \mathrm{k}$ detector with $12 \mu \mathrm{m}$ pixels, resulting in a roughly $49 \times 49 \mathrm{~mm}$ imaging field.

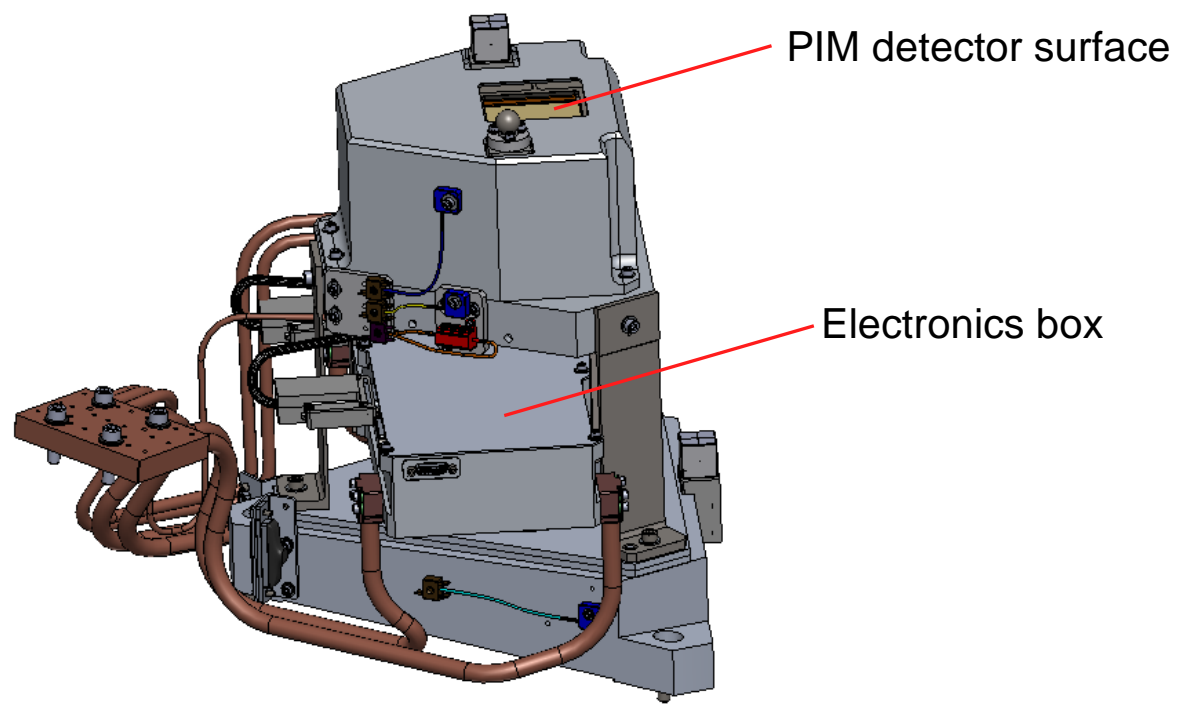

Figure 2-3 The Pupil Imaging Module (PIM).

\subsection{SI hardware}

As mentioned above, each SI contains a reflective PAR that is, or can be inserted, into its pupil plane. Schematics of the SI PAR's are shown in Figure 2-4. They are not shown at the same scale - they range in size from a few mm to tens of $\mathrm{mm}$ in diameter. Like the OSIM PAR's, the SI PAR's also have some as-built offset between their internal PAR and pupil locations that must be taken into account. These offsets were measured by the SI teams prior to their instrument deliveries. A summary of SI nominal offsets is shown in Table 2-3. 


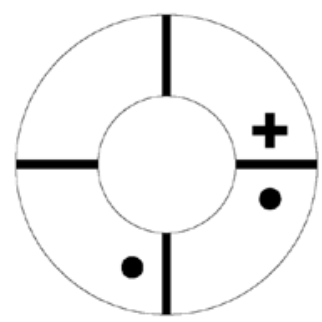

a) Guider

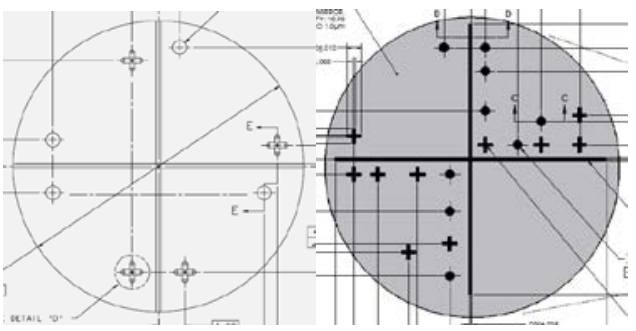

b) NIRISS

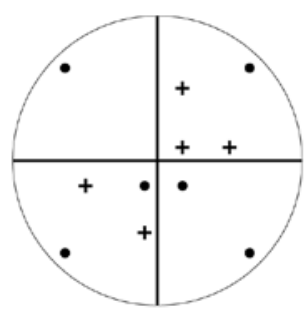

d) NIRSpec

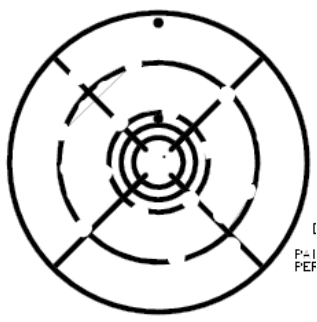

e) NIRCam

Figure 2-4 Schematics of the SI PAR's. They are not shown at the same scale - they range in size from a few mm to tens of $\mathrm{mm}$ in diameter.

Table 2-3 Summary of nominal SI expected PAR-pupil offsets, based on SI calibrations prior to delivery to ISIM.

\begin{tabular}{|c|c|c|c|c|c|c|}
\hline & \multicolumn{3}{|c|}{ PAR-pupil offsets } & \multicolumn{3}{c|}{ 1 $\mathbf{c}$ error } \\
\hline SI & V2 (\%) & V3 (\%) & Roll (mR) & V2 (\%) & V3 (\%) & Roll (mR) \\
\hline FGS Guider & 0.40 & -1.02 & 15.95 & 0.08 & 0.08 & 1.47 \\
\hline NIRISS* & -0.18 & -2.76 & 19.53 & 0.13 & 0.06 & 2.82 \\
\hline MIRI & -2.97 & 1.55 & -82.55 & 0.13 & 0.15 & 2.47 \\
\hline NIRSpec & 0.02 & -0.06 & 0.00 & 0.10 & 0.10 & 4.36 \\
\hline NIRCam A & -1.01 & 0.88 & 49.76 & 0.07 & 0.09 & 1.21 \\
\hline NIRCam B & 0.58 & 0.18 & -25.16 & 0.07 & 0.09 & 1.03 \\
\hline
\end{tabular}

* Internal mechanism settings for NIRISS can affect the expected pupil offset. The values shown are based on the final settings as of the end of CV3.

Together, the OSIM and SI offsets provide an 'expected pupil shear' for each SI. That is, even if the pupil were aligned perfectly we would still expect some as-built shear to show up due to the as-built values above. This expected shear must be subtracted from any measurement to get an accurate pupil shear measurement. 


\section{DATA CAPTURE AND ANALYSIS}

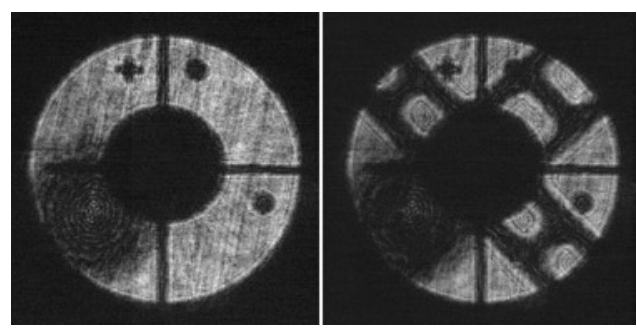

a) Guider

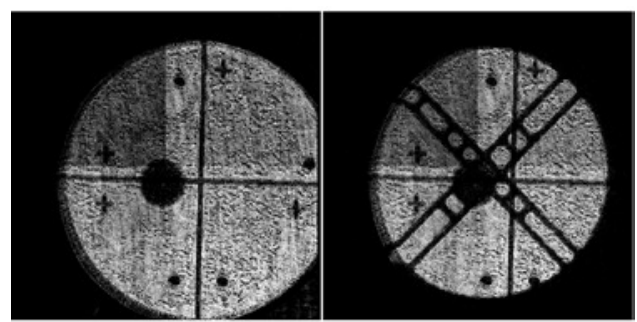

b) NIRISS

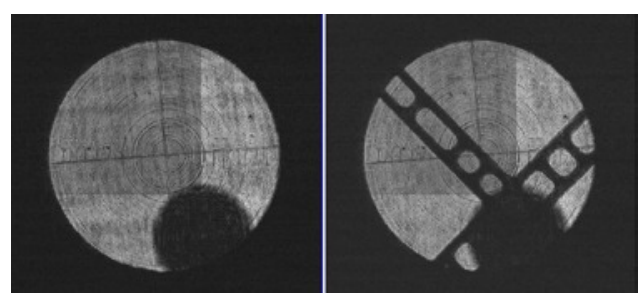

c) MIRI

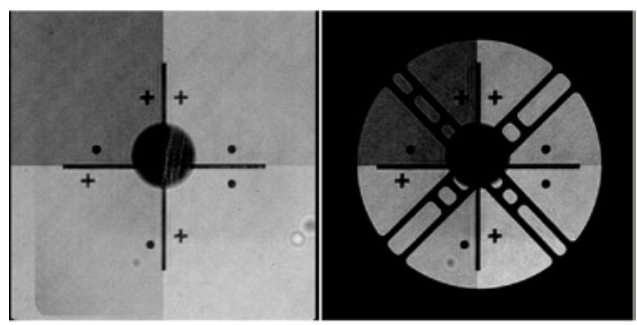

d) NIRSpec
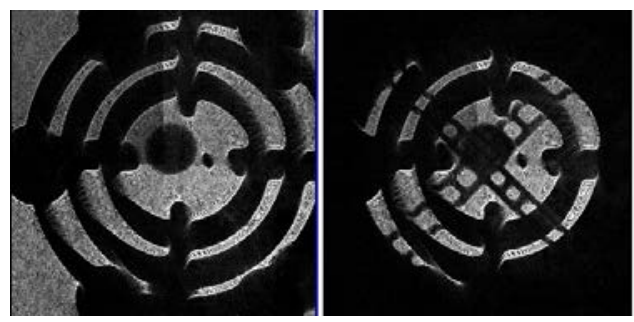

e) NIRCam A (NIRCam B is similar)

Figure 3-1 PIM images of the SI PARS. On the left is an image with the OPEN OSIM mask; on the right are images with the OSIM PAR1 mask. The images are not shown at the same scale - some have been enlarged to show detail.
Each of the SIs has a specific field point that is used for pupil alignment tests. To perform a pupil measurement, OSIM is configured to point at that field point with one of the OSIM PARs used in place of the tricontagon. The $0.640 \mu \mathrm{m}$ laser diode source is chosen for PAR testing, because FM3 is a beam splitter at that wavelength. Source power and PIM exposure settings are chosen to give the shortest exposures possible. Since the PAR test results in such a large image on the PIM detector (i.e., not an in-focus point image) and the wavelength used gets attenuated by FM3, the source used for the PIM testing is generally much higher power than those used for other tests. With the exception of the FGS Guider, putting the SI into the proper configuration for the test will also close off and protect the SI detectors from the higher source power used. While the NIRISS Dual Wheel positions for PAR testing do not completely close off the detector, it will sufficiently attenuate the PAR source.

Figure 3-1 shows PIM images from each SI. The images on the left were taken with the OPEN OSIM aperture to show what the SI PAR looks like on its own. The images on the right were taken with PAR1 in place. The images are not shown at the same scale. Also visible in the images, particularly Figure 3-1(d), is the difference in responses between the four quadrants of the PIM detector. The checkerboard effect had no effect on pupil shear measurements.

After the PIM images are captured, the targets in the image are compared against PAR templates. The templates were created by putting the as-built PAR's into CODEV and tracing them through the system. For example, the as-built OSIM PAR's were entered into the optical model as a set of obscurations at the aperture stop. Rays are aimed at the corners of these obscurations, and traced all the way through the system - including the SI - to the PIM surface. In this manner, any distortions or rotations caused by the OSIM optical system (such as FM3) or rotations caused by beam steering are automatically taken into account and included in the template.

The instrument PAR templates were created similarly. The PAR designs were included in their optical systems and rays were traced to transfer their dimensions to the PIM surface. Figure 3-2 contains an example of the NIRSpec image with the OSIM and SI templates overlaid.

The blue lines belong to the SI PAR template, and is positioned and rotated by eye to match the SI PAR shadow. The red lines, belonging to the OSIM PAR, are positioned to match the shadow of the OSIM PAR. The "measured image shear" is then the distance between the centers of the two templates. This is not the final pupil shear, only the shear that is seen in the PIM images. 


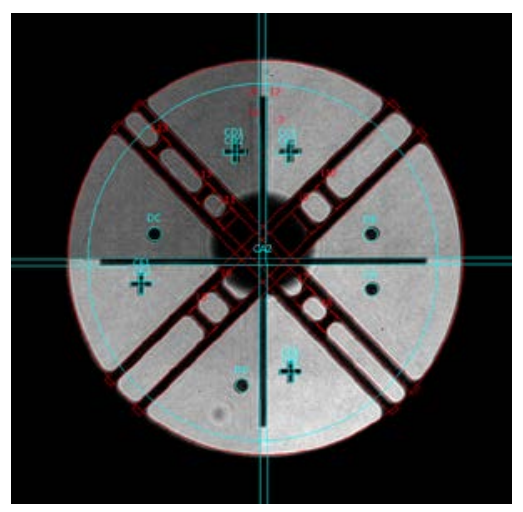

Figure 3-2 Example of a PIM image with the OSIM and SI (NIRSpec) templates overlaid.

Pupil shear measurements were made at several points in the ISIM test campaign to track pupil motion over time. For brevity, the measured image shear for only the final CV3 shear measurements are shown in Table 3-1. Uncertainties in this analysis are based on $0.012 \mu \mathrm{m}$ pixels in the PIM detector. The measurements were assumed to be within 4 pixels, or about $0.011 \% 1 \sigma$. A similar reasoning was used for the roll measurements, where $0.5^{\circ} 3 \sigma$ uncertainty was used for both the SI and OSIM PAR's. The uncertainty in the final measurement then comes out to about $10.07 \mathrm{mR} 1 \sigma$ uncertainty. Note that the $1 \sigma$ pupil roll uncertainty being carried is larger than the roll requirement given in Section 1 , and dominates the roll uncertainty in the final pupil shear results.

Table 3-1 Summary of measured image shears in the final CV3 pupil shear measurements.

\begin{tabular}{|c|c|c|c|c|c|c|}
\hline & \multicolumn{3}{|c|}{ Image Offset } & \multicolumn{3}{c|}{ 1 0 error } \\
\hline SI & V2 (\%) & V3 (\%) & Roll (mR) & V2 (\%) & V3 (\%) & Roll (mR) \\
\hline FGS Guider & -0.14 & -0.35 & -3.32 & 0.011 & 0.011 & 10.07 \\
\hline NIRISS & 1.32 & -3.21 & 12.39 & 0.011 & 0.011 & 10.07 \\
\hline MIRI & -3.97 & 2.28 & -97.56 & 0.011 & 0.011 & 10.07 \\
\hline NIRSpec & 0.22 & -0.04 & -21.12 & 0.011 & 0.011 & 10.07 \\
\hline NIRCam A (PAR) & -0.82 & -1.20 & 63.88 & 0.052 & 0.052 & 14.24 \\
\hline NIRCam B (PAR) & -0.44 & -2.21 & -72.33 & 0.052 & 0.052 & 14.24 \\
\hline
\end{tabular}

To calculate the final pupil shear, the expected pupil shears shown in Table 2-2 and Table 2-3 must be subtracted from the measured image shear above. The final pupil shears, based on PAR images, are shown in Table 3-2.

The uncertainties shown above for NIRCam are higher than the rest due to the distorted nature of the NIRCam PIM images as shown in Figure 3-1(e) - lines that should be straight are wavy, curved, etc. The distortion was likely due to the process used to fabricate the NIRCam PAR's. For this reason, an accurate measure of pupil shear and roll weren't possible with the same uncertainties as the other SI's. Fortunately, the NIRCam modules are equipped with a Pupil Image Lens (PIL) that can be used to directly view both the OSIM and SI apertures.

PIL images of the OSIM tricontagon were compared against images of the NIRCAM Dispersed Hartmann Sensor (DHS) apertures to measure pupil shear directly. In this case, the measured image shear is the pupil shear, however, the OSIM uncertainties still come into play. Images of the OSIM tricontagon and one of the DHS's is shown in Figure 3-3. The measured pupil shear from the PIL images is included in Table 3-2. 

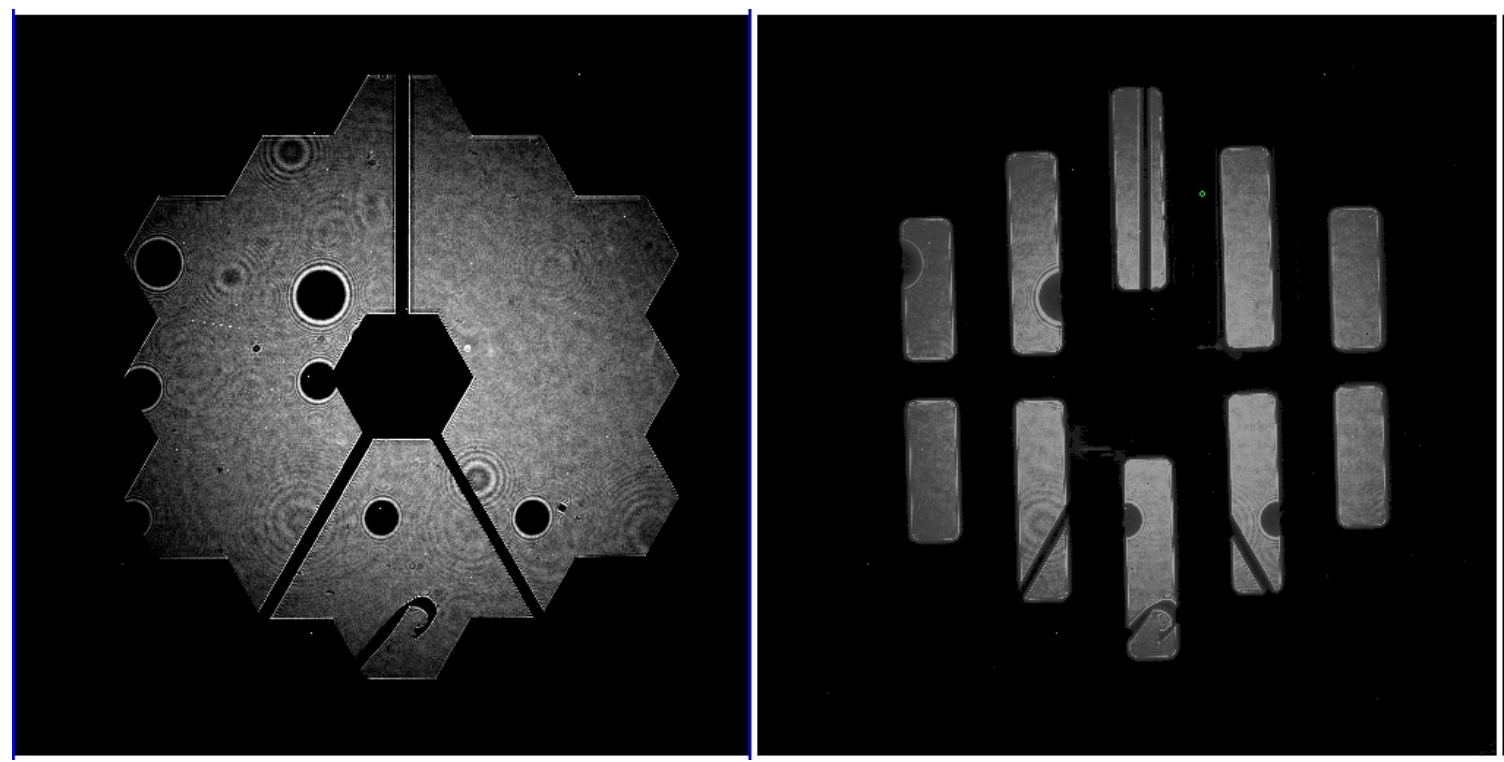

Figure 3-3 NIRCam PIL images of the OSIM tricontagon and one of the DHS's. NIRCam PIL images such as these were used to calculate a better estimate of pupil shear in the NIRCam modules.

Table 3-2 Summary of pupil shear in the final CV3 pupil shear measurements. These are the measured image shears with the expected pupil shears subtracted.

\begin{tabular}{|c|c|c|c|c|c|c|}
\hline & \multicolumn{3}{|c|}{ Pupil shear } & \multicolumn{3}{c|}{ 1 $\boldsymbol{0}$ error } \\
\hline SI & V2 (\%) & V3 (\%) & Roll (mR) & V2 (\%) & V3 (\%) & Roll (mR) \\
\hline FGS Guider & -0.54 & 0.72 & -27.62 & 0.29 & 0.29 & 10.19 \\
\hline NIRISS & 1.51 & -0.41 & -2.95 & 0.31 & 0.29 & 10.47 \\
\hline MIRI & -1.00 & 0.78 & -10.78 & 0.31 & 0.32 & 10.39 \\
\hline NIRSpec & 0.21 & 0.06 & -16.77 & 0.30 & 0.30 & 10.99 \\
\hline NIRCam A (PAR) & 0.20 & -2.04 & 18.32 & 0.30 & 0.30 & 14.30 \\
\hline NIRCam B (PAR) & -1.01 & -2.35 & -42.91 & 0.30 & 0.30 & 14.30 \\
\hline NIRCam A (PIL) & -0.11 & 0.08 & -30.93 & 0.28 & 0.28 & 10.08 \\
\hline NIRCam B (PIL) & 0.00 & 0.00 & 33.59 & 0.28 & 0.28 & 10.08 \\
\hline
\end{tabular}

\subsection{On-orbit predictions}

Structural-Thermal-Optical (STOP) analysis was used to predict the on-orbit change in pupil shear due to the absence of gravity. The STOP-predicted values were added to the values in Table 3-2, and the resulting on-orbit predictions are shown in Table 3-3. The NIRCam values do not change with gravity release because NIRCam will undergo an on-orbit pupil alignment. 
Table 3-3 On-orbit pupil shear predictions based on STOP analysis and the measured pupil shears shown in Table 3-2.

\begin{tabular}{|c|c|c|c|c|}
\hline & \multicolumn{4}{|c|}{ On-orbit pupil shear predictions } \\
\hline SI & V2 (\%) & V3 (\%) & Rad (\%) & Roll (mR) \\
\hline FGS Guider & -0.10 & 1.00 & 1.00 & -27.64 \\
\hline NIRISS & 1.92 & -0.18 & 1.93 & -2.90 \\
\hline MIRI & -1.20 & 0.96 & 1.54 & -10.68 \\
\hline NIRSpec & 0.64 & -0.34 & 0.72 & -16.77 \\
\hline NIRCam A (PAR) & 0.20 & -2.04 & 2.05 & 18.32 \\
\hline NIRCam B (PAR) & -1.01 & -2.35 & 2.55 & -42.91 \\
\hline NIRCam A (PIL) & -0.11 & 0.08 & 0.13 & -30.93 \\
\hline NIRCam B (PIL) & 0.00 & 0.00 & 0.00 & 33.59 \\
\hline
\end{tabular}

\subsection{Direct comparison of pupil images}

Overlaying pupil images from different PAR measurements allows a direct comparison of pupil motion over time. For example, pupil images from the CV2 Final Optical Baseline and CV3 Optical Baseline tests can be compared to show pupil motion between thermal-vacuum cycles, before and after SI rework, ISIM-level vibration, and/or acoustics exposure. This is a simplistic approach, which only compares one point in each image.

Some of the CV2 images suffer from uneven pupil illumination that made it difficult to obtain even contrast between images. This was due to an issue with the PIM system, not the flight instruments. Also, since NIRCam and NIRISS have mechanisms that affect the pupil alignment, and those mechanisms were exercised, this cannot be seen as a measure of pupil stability.

Still, despite the shortcomings of this comparison, it provides a useful cross-check for the pupil analysis routines.

Overlaid Pupil images from CV2 FOB and CV3 OB are shown in Figure 3-4. The left image in each group is the original PIM image. The area outlined in blue is magnified on the right. The faint white line (which is hard to see) in the magnified image shows the magnitude and direction of the shift. The red images are from CV2 ('before') and the green are from CV3 ('after').

Images from the NIRISS and NIRSpec tests suffer from particularly uneven response at the PIM. Adjusting for the best contrast in the magnified images made the full images appear red on top and green on the bottom. As the magnified images show, however, they are complete images of the PARs

The results of this comparison are shown in Table 3-3. Uncertainties on the pupil shear differences derived from the overlaid images were not calculated, but the differences between the image deltas and the deltas from the PAR analysis are all under the roughly $0.3 \%$ uncertainties in the PAR analysis. 


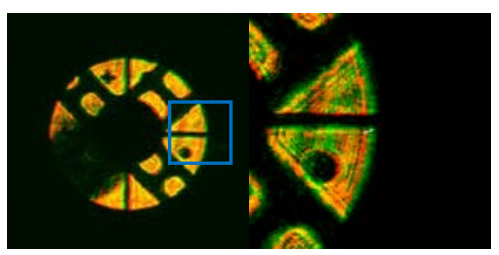

a) Guider

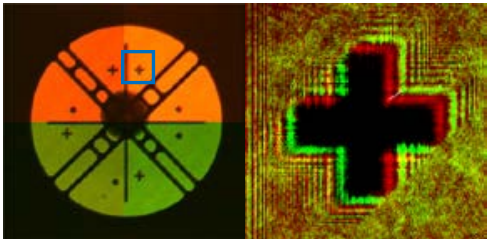

d) NIRSpec

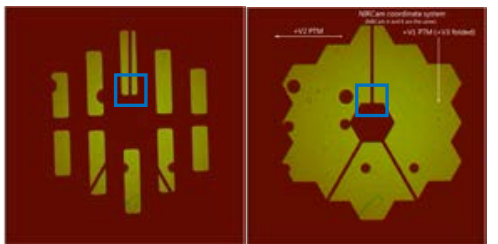

g) DHS and tricontagon (PIL)

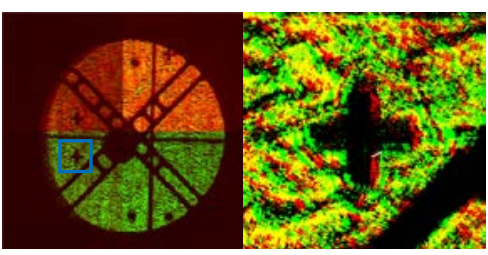

b) NIRISS

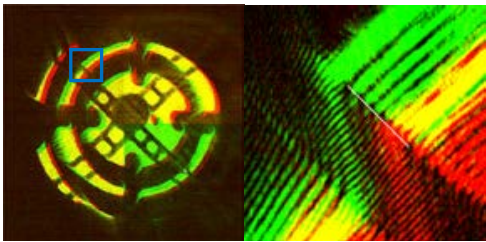

e) NIRCam A (PAR)

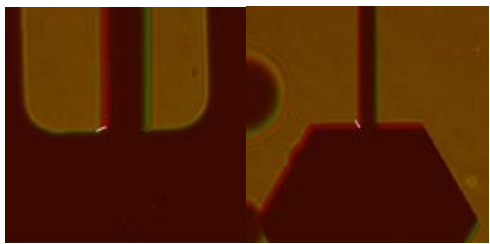

h) NIRCam A (PIL)

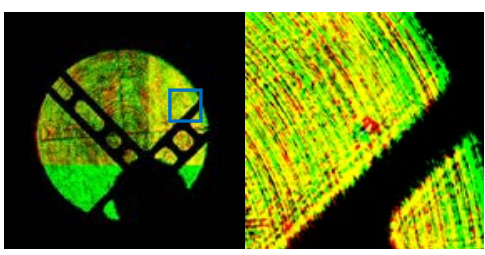

c) MIRI

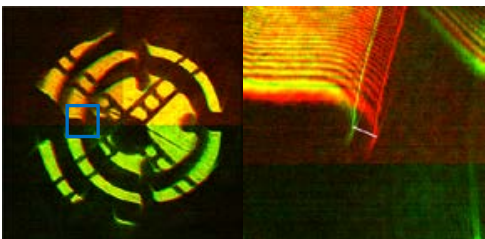

f) NIRCam B (PAR)

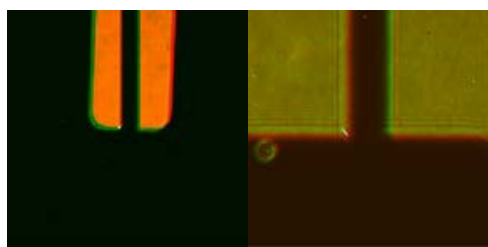

i) NIRCam B (PIL)

Figure 3-4 Overlaid pupil images from CV2 (red) and CV3 (green)

Table 3-4 Difference between CV2 and CV3 images as seen by direct comparison and through full PIM analysis.

\begin{tabular}{|c|c|c|c|c|}
\hline & \multicolumn{2}{|c|}{$\begin{array}{c}\text { Delta from } \\
\text { direct comparison }\end{array}$} & \multicolumn{2}{c|}{$\begin{array}{c}\text { Delta from } \\
\text { full PIM analysis }\end{array}$} \\
\hline SI & V2 (\%) & V3 (\%) & V2 (\%) & V3 (\%) \\
\hline FGS Guider & -0.08 & -0.19 & -0.1 & -0.15 \\
\hline NIRISS & -0.07 & -0.17 & 0.02 & -0.26 \\
\hline MIRI & -0.14 & -0.15 & -0.11 & -0.15 \\
\hline NIRSpec & -0.1 & -0.15 & -0.17 & -0.16 \\
\hline NIRCam A (PAR) & -0.46 & 0.46 & -0.32 & 0.59 \\
\hline NIRCam B (PAR) & -0.14 & 0.33 & -0.12 & 0.31 \\
\hline NIRCam A (PIL) & 0.00 & 0.72 & -0.2 & 0.81 \\
\hline NIRCam B (PIL) & 0.05 & 0.56 & -0.14 & 0.65 \\
\hline
\end{tabular}




\section{CONCLUSIONS}

The PIM and PAR system was used to record ISIM pupil shear measurements at several points during ISIM cryogenicvacuum cycles CV1RR through CV3. The PIM system was adequate in making lateral pupil shear measurements, but uncertainty in pupil roll using this method was too high to make measurements to the level of the requirement.

The PAR measurements over time are shown in Figure 4-1. The colored circles represent the measurement uncertainties in the final measurements. The 'tail' of each circle shows the pupil motion over time. The pupil roll results are shown in Figure 4-2. Uncertainties are shown as wedges. The height of the wedges in Figure 4-2 have no meaning. They were adjusted just to make the wedges easier to parse. NIRCam and NIRSpec were not present for the CV1RR test, so results for those two SI's begin with CV2. A more complete uncertainty estimate, including systematic factors, is provided by Rohrbach et $\mathrm{al}^{4}$.

All of the lateral pupil shears are under the required 3.1\% for ISIM - even for the distorted NIRCam PAR images. The roll values, however, are all outside the requirement with the exception of NIRISS. When measurement uncertainties are included, however, NIRISS is out of specification as well, especially since only $1 \mathrm{~s}$ values are shown. In light of this, we have completed an analysis of the worst-case shear and roll alignment conditions for the various instrument modes with performance that depends on roll alignment. We find that the as-built alignment provides acceptable performance ${ }^{4}$.

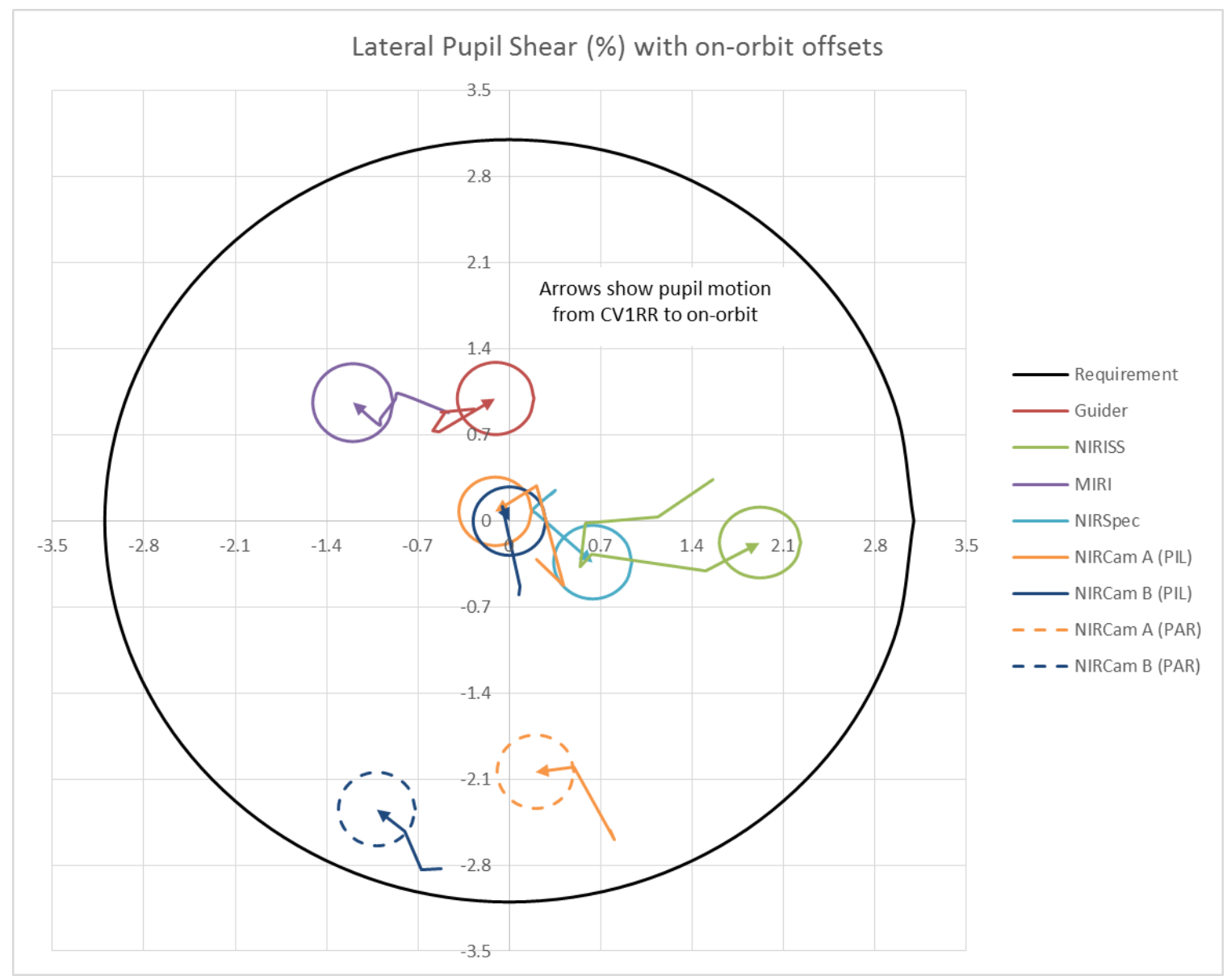

Figure 4-1 Pupil shear measurements over time. Pupil motion from CV1RR through CV3 and on-orbit predictions are shown. The circles show the $1 \sigma$ measurement uncertainty. The line (and arrow) show the pupil motion over time. 


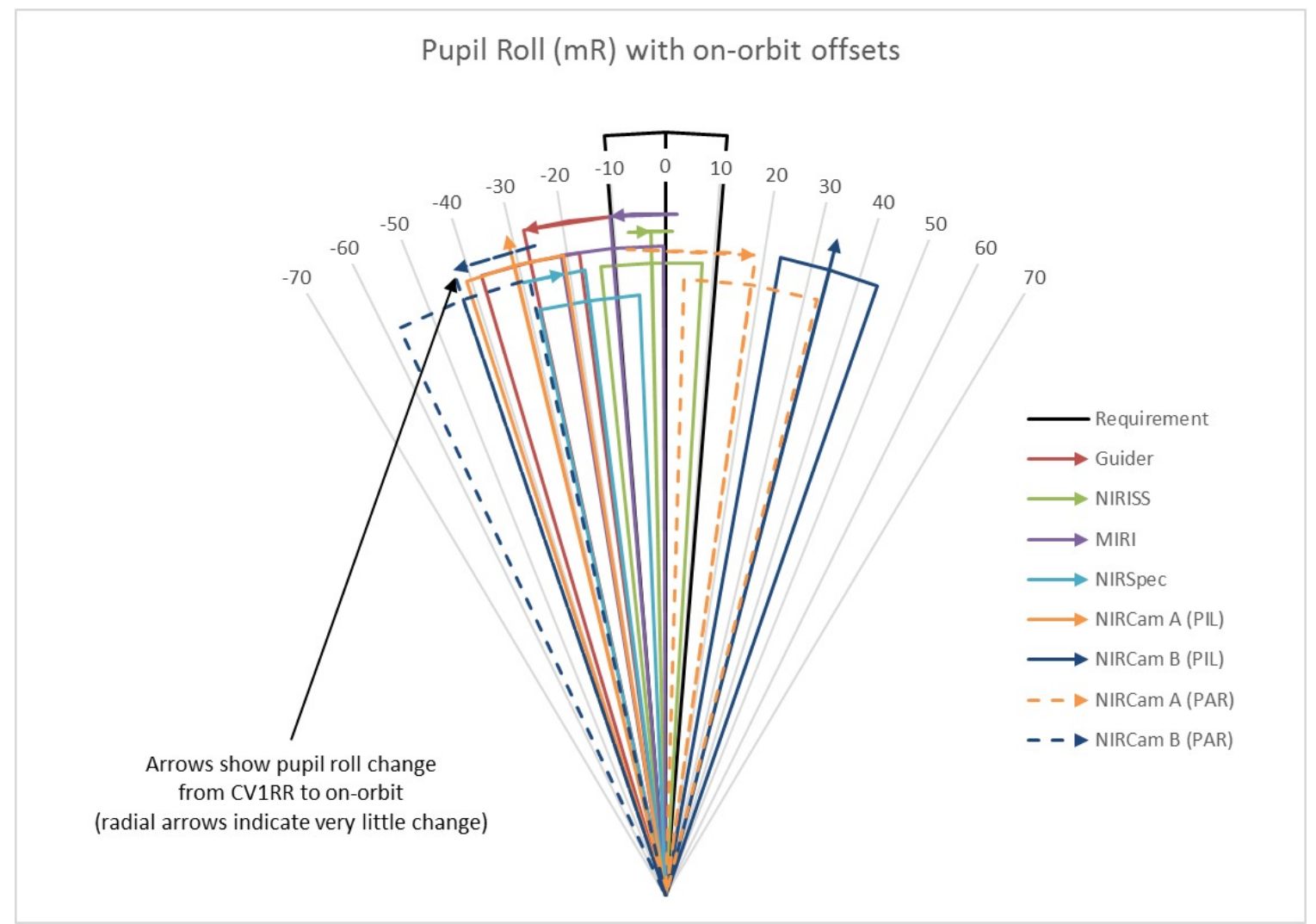

Figure 4-2 Pupil roll measurements made over time. $1 \sigma$ uncertainties are shown as wedges around the final roll value.

\section{ACKNOWLEDGEMENTS}

The authors gratefully acknowledge the contributions of optical, mechanical, electrical, and systems engineers, managers, and scientists associated with the JWST project, the ISIM element, the Science Instruments and their partners [i.e., FGS Guider and NIRISS, provided by the Canadian Space Agency (CSA) and Honeywell; MIRI, provided by the European Consortium with the European Space Agency (ESA), and by the NASA Jet Propulsion Laboratory (JPL); NIRCam, provided by the University of Arizona and Lockheed Martin; and NIRSpec, provided by ESA, with components provided by GSFC], and OSIM.

We acknowledge the leadership of P. Davila (GSFC, retired), J. Hagopian (GSFC) and B. Bos (GSFC) in the development of requirements, their organization, and the TPT effort and strong support from J. Dunn (GSFC).

We dedicate this paper to the memory of B. Greeley. His many contributions to the development of OSIM and ISIM were vital to our success.

This work is supported by the James Webb Space Telescope project and NASA Goddard Space Flight Center. 


\section{REFERENCES}

[1] Bos, B. J., Davila, P. S., Jurotich, M., Hobbs, G., Lightsey, P., Contreras, J. and Whitman, T., “The James Webb Space Telescope instrument suite layout: optical system engineering considerations for a large, deployable space telescope," Proc. SPIE 5487, 734-745 (2004).

[2] Bos, B. J., Kubalak, D. A., Antonille, S. R., Ohl, R. G., Hagopian, J. G., Davila, P. S., Sullivan, J., Sánchez, M., Sabatke, D., Woodruff, R. A., te Plate, M., Evans, C., Isbrucker, V., Somerstein, S., Wells, M. and Ronayette, S., "Cryogenic pupil alignment test architecture for the James Webb Space Telescope integrated science instrument module,” Proc. SPIE 7010, 70103C (2008).

[3] Maszkiewicz, M., et al, "FGS/NIRISS on James Webb Space Telescope - Pupil Alignment Methodology and Metrology,” International Conference on Space Optics Proceedings, in press (2016).

[4] Rohrbach S., et al, "Critical science instrument alignment of the James Webb Space Telescope (JWST) Integrated Science Instrument Module (ISIM),” Proc. SPIE 9951, this volume (2016). 